\title{
An influence of low-stability region on dense gas phenomena and their peculiarities in the ORC fluids
}

\author{
Dominika Matuszewska $^{1}$, Karol Sztekler ${ }^{1}$, Jan Gorski ${ }^{1, a}$ \\ ${ }^{1}$ AGH University of Science and Technology, Faculty of Energy and Fuels, 30-059 Krakow, Poland
}

\begin{abstract}
An existence of low stability region in the dense vapours and its influence on some peculiarities in behaviour of selected dry and isentropic ORC fluids is discussed. The retrograde phenomena in the flow of BZT fluids [1.] can be simply related to the mechanical and thermodynamic stability parameters. These new refrigerant and their properties have been analysed based on the software tools REFPROP v.9.1 [2.]. Test examples have confirmed an importance of low thermodynamic stability area in the vicinity of saturation boundary line and neighbourhood of critical point of the fluid. The analytical results have been obtained for selected pure fluids applicable in the ORC and heat pump technology such $\mathrm{C}_{4} \mathrm{H}_{10}, \mathrm{C}_{6} \mathrm{H}_{5} \mathrm{CH}_{3}, \mathrm{C}_{12} \mathrm{H}_{26}, \mathrm{R} 123$, R134a, R227ea, R245fa, low GWP hydrofluoroolefins (R1234xxX)and a group of linear and cyclic siloxanes.
\end{abstract}

\section{Introduction}

\subsection{Organic Rankine Cycle}

In our society the demand for electric and mechanical energy is still growing up. Besides the renewable energy sources, such wind, hydropower and photovoltaic, most of our energy is being generated from the thermal energy sources. Depending on the application, different thermodynamic cycles have been developed in practice. Commonly used combustion engines, steam turbines and gas turbines always use high temperature heat sources. Still a lot of waste heat is being released in these cycles often on relatively high temperatures. In case this waste heat can be recovered usefully in the CHP installation at a high fuel utilization ratio. Even low temperature industrial waste heat, that can't be recovered otherwise, could still have a practical application in this way. A suitable thermodynamic cycle to this purpose is the Organic Rankine Cycle (ORC), [1-4]. This cycle resembles the classical steam cycle commonly used in thermal power stations. If only a heat source on low temperature is available or in small scale applications, it could be advantageous to replace water/steam by the suitable organic medium. Mainly organic fluids, like refrigerants, toluene, or silicone oils are used. These fluids are characterized as 'dry' or 'isentropic fluids' and have some interesting properties comparing to water and steam, [1, 2].

Figure 1 shows the T-s diagram for an ideal organic Rankine cycle using toluene as the working medium operating in the range of temperatures $325 \div 500 \mathrm{~K}$. Due concave shape of right saturation curve in the vapour dome, the turbine outlet parameters lies in the superheated region. This feature of the organic fluids and other high-molecular mass fluids completely eliminates concerns about turbine blade erosion.

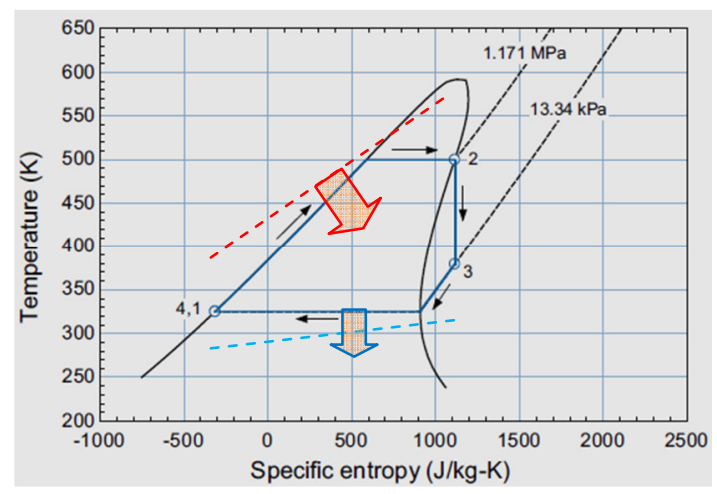

Fig. 1.Subcritical ORC cycle for toluene.

Due to characteristics of dry fluids, the vapour remains largely superheated after expansion (see, Fig.1, point 3) and the regeneration in the ORC system is sometimes necessary to improve its efficiency. Therefore, ORC system can be effectively applied to waste heat recovery in the exhaust gases, and also a higher net electrical capacity can be achieved with an ORC compared to a typical steam power cycle. However, several additional questions exist when we try to use some new fluids in the ORC power system. Firstly, an important problem is the lack of complete data on its thermal and transport properties. Secondly, the material compatibility, toxicity

\footnotetext{
${ }^{\mathrm{a} C}$ Corresponding author: jagorski@agh.edu.pl
} 
or flammability and the environmental safety problems will be limiting for their applications [1.]

\subsection{Characteristics of ORC fluids}

The organic fluids in an ORC system can be used at a much lower evaporation temperature and pressure and its superheating is not required. Their evaporation heat is also small compared to the water. This results in less higher demands to the temperature level of the heat source. Many recently used working fluids in the ORC and heat pump operation are still not satisfactory from the point of view the compatibility of materials or security for the human health, biological life and the environment. Typical hydrocarbons (HC's) and its derivative (HFC, HCFC refrigerants) are popular as low0boiling point media in the technical applications. A lot of existing on the market pure fluids and mixtures will be in the nearest future replaced by new more suitable fluids. A great attempt has been made to develop the harmful groups of substances such hydrofluoroolefins (HFO's), siloxanes and the nearly-azeotropic fluid mixtures [1-5]. For our analysis purposes we have selected fifteen fluids from these groups, see Table 1. All fluids are arranged on the basis of their molecular mass.

Table 1. Basic properties of the selected ORC fluids [2.].

\begin{tabular}{|c|c|c|c|c|c|}
\hline Fluid & $\begin{array}{c}\text { Molar } \\
\text { mass } \\
\text { M g/mol }\end{array}$ & $\begin{array}{c}\text { Acentric } \\
\text { Factor } \\
\boldsymbol{\omega}\end{array}$ & $\begin{array}{c}\text { NBP } \\
\mathrm{K}\end{array}$ & $T_{c} \mathrm{~K}$ & $P_{c} \mathrm{MPa}$ \\
\hline Butane & 58.12 & 0.201 & 273.0 & 425.1 & 3.796 \\
\hline Toluene & 92.14 & 0.266 & 383.8 & 591.8 & 4.126 \\
\hline R134a & 102.0 & 0.327 & 247.1 & 374.2 & 4.059 \\
\hline R1234yf & 114.0 & 0.276 & 243.7 & 367.9 & 3.382 \\
\hline R1234zeZ & 114.0 & 0.327 & 282.9 & 423.3 & 3.533 \\
\hline R1234zeE & 114.0 & 0.313 & 254.2 & 382.5 & 3.635 \\
\hline R245fa & 134.1 & 0.378 & 288.3 & 427.2 & 3.651 \\
\hline R123 & 152.9 & 0.282 & 301.0 & 456.8 & 3.662 \\
\hline $\begin{array}{c}\text { Siloxane } \\
\text { MM }\end{array}$ & 162.4 & 0.418 & 373.4 & 518.8 & 1.939 \\
\hline R227ea & 170.0 & 0.357 & 256.8 & 374.9 & 2.925 \\
\hline Dodecane & 170.3 & 0.574 & 489.3 & 658.1 & 1.817 \\
\hline $\begin{array}{c}\text { Siloxane } \\
\text { MDM }\end{array}$ & 263.5 & 0.529 & 425.7 & 564.1 & 1.415 \\
\hline $\begin{array}{c}\text { Siloxane } \\
\text { D4 }\end{array}$ & 296.6 & 0.592 & 448.5 & 586.5 & 1.332 \\
\hline $\begin{array}{c}\text { Siloxane } \\
\text { MD2M }\end{array}$ & 310.7 & 0.668 & 467.5 & 599.4 & 1.227 \\
\hline $\begin{array}{c}\text { Siloxane } \\
\text { D5 }\end{array}$ & 370.8 & 0.658 & 484.1 & 619.2 & 1.160 \\
\hline $\begin{array}{c}\text { Siloxane } \\
\text { D6 }\end{array}$ & 444.9 & 0.736 & 518.1 & 645.8 & 0.961 \\
\hline $\begin{array}{c}\text { Siloxane } \\
\text { MD4M }\end{array}$ & 459.0 & 0.825 & 533.9 & 653.2 & 0.877 \\
\hline
\end{tabular}

Based on the data collected in Table 1, there are two groups of test fluids - hydrocarbons and refrigerants with the low-boiling point (NBP), close to the ambient temperature and a group of high-boilng point of siloxanes and two HC's (toluene and dodecane). All of above mentioned fluids can exhibit complex phenomena during the thermal-flow processes which take place in the turbomachinery, heat exchangers and the pipeline components.

\section{Similarity parameters in the flow of dense gas and vapour}

\subsection{Problem issues}

There are several aspects of an analysis of the compressible fluid behaviour which can be usually focused on the following questions:

- how to categorise different working fluids respect to their peculiarities in the thermal-flow processes,

- which form of data presentation (EOS) is preferable considering its consistency, quality and usability,

- is it possible to adopt any form of an EOS to the gas dynamics problems and simply express basic relations in the flow of dense vapour and gas,

- what are the similarity criteria in the dense gas flow and how correspond to an ideal gas model,

These questions are still open but now we can observe a great progress in development of more effective tools for analysing of expansion and compression processes in various fluids including refrigerants, natural and organic fluids. Some typical measures such heat capacity ratio and isentropic index are insufficient for presentation the change of real fluid and flow parameters. It is necessary to use more consistent approach, applicable both in a basic thermal-flow balances and the CFD numerical schemes [5,7].

Dense gas flow phenomena are still not well-recognised and its investigation, interpreting and mathematical backgrounds is an important part of many research institutions and industry [1-5,7]. It is caused by rapid growth of interesting in utilization of renewable and waste heat energy resources including ORC and heat pumps. It is visible, see Table 1, that according to the growth of molecular mass the value of acentric factor $\omega$ is also higher. This measure of fluid complexity directly relates to many important parameters characterising a particular fluid behaviour $[5,7,8]$.

\subsection{Saturation line behaviour of ORC fluids}

Heavy fluids with the composed molecular structure exhibit more frequently non-classical behaviour in vicinity of saturated vapour line $(x=1)$. This curve is often a positive slope on the $T$-s plane, as opposed to the water and ammonia. This category of fluids is called 'dry' and 'isentropic fluids' when this slope is zero [4], see Fig. 2. These geometric features inside the VLE 
domainare verycharacteristicin the case of typical lowboiling fluids with molecular mass exceeding $100 \mathrm{~g} / \mathrm{mol}$. Other important consequence of application of the 'dry' or 'isentropic' fluids is an influence of real gas effects on the cycle performance parameters (produced power and efficiency) [2,7].

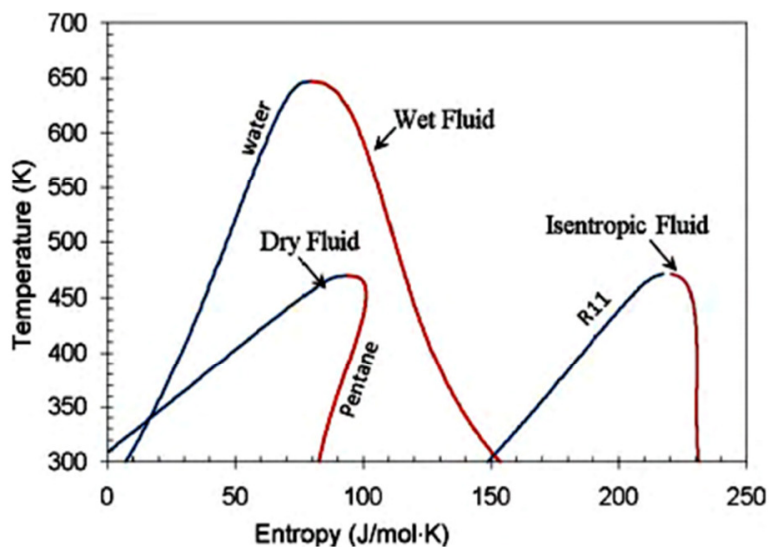

Fig.2. Wet, isentropic and dry fluids [5].

Therefore, a direct information on the shape of right vapour-liquid (V-L) coexistence line gives a value of the derivative $\sigma=(d s / d T)_{\mathrm{x}=1}$. This molecular complexity parameter $\sigma$ is usually referred to the reduced temperature $T_{r}=0.7$ and defined as $[2-5]$

$$
\begin{gathered}
\sigma_{T r=0.7}=\frac{T_{c}}{R}\left(\frac{d s}{d T}\right)_{x=1}= \\
=\frac{T_{c}}{R}\left[\left(\frac{\partial s}{\partial p}\right)_{T}\left(\frac{d p}{d T}\right)_{x=1}+\left(\frac{\partial s}{\partial T}\right)_{p}\right]_{T r=0.7}
\end{gathered}
$$

The simplified form of eq. (1) is frequently given as [5]

$$
\sigma_{T r=0.7}=\frac{T_{c}}{R}\left[\frac{c_{p}^{0}}{T}-\frac{R}{p}\left(\frac{d p}{d T}\right)_{x=1}\right]_{T r=0.7}
$$

An equivalent formulation can be introduced by using the 'retrogradicity' parameter proposed by Lambrakis, [11]

$$
\tilde{r}_{\sigma}=\left(\frac{\partial T}{\partial v}\right)_{p}\left(\frac{d s}{d p}\right)_{x=1} \geq 0
$$

This parameter is a measure of departure from the normal fluids and is important when we analyse a supercritical and high-pressure subcritical ORC cycle. In such cases the process in the expander take place in a real-gas area and all physical constants significantly vary with temperature and pressure. It can also partially take place in two-phase region, but finally the fluid undergoes of the 'drying process'. From the well-known thermodynamic identities equation (3) takes the form [11]

$$
\tilde{r}_{\sigma}=\left(\frac{c_{p}^{\prime \prime} T}{\Delta h_{l v}}\right)\left(\frac{z_{v T}}{z_{T v}}\right)^{\prime \prime}\left(1-\frac{v^{\prime}}{v^{\prime \prime}}\right)-1 \geq 0
$$

where indices (") are related to the quantities on the right vapour-liquid line and (') to parameters on the left curve. The additional parameters $z_{T v}$ and $z_{v T}$ are two independent the VCD functions [11], see Appendix.

The first term in the eq. (4) is the reciprocal of Jacob's Number $J a$, which is always positive and greater than one, but the second, ratio $z_{v T} / z_{T v}$, is less than one. In the limiting case the product of these two terms can achieve value close to unity. Ultimately, the retrogradicity parameter can be less than zero because of the specific volume ratio $v \nu^{\prime \prime}$ is negligible at the sub-critical pressure area.

While studying any real system of fluid properties and models, it is necessary take into consideration whether they respond to the stability conditions. These conditions have great importance for a fluid behaviour in the dense gas region, close to saturation line and the critical point. The simple expression of the thermodynamic stability condition for one-component homogeneous $p v T$ system is given [9]

$$
D=\frac{\partial(T,-p)}{\partial(s, v)}=\left|\begin{array}{ll}
(\partial T / \partial s)_{v} & (\partial T / \partial v)_{s} \\
(\partial T / \partial v)_{s} & (\partial p / \partial v)_{s}
\end{array}\right| \geq 0
$$

After simple manipulations one obtains

$$
D=-\left(\frac{\partial p}{\partial v}\right)_{S}\left(\frac{\partial T}{\partial s}\right)_{p}=-\frac{T}{c_{v}}\left(\frac{\partial p}{\partial v}\right)_{T}
$$

It can be simply written in the reduced form [11]

$$
\bar{D}=D\left(\frac{v_{c}}{T_{c}}\right)^{2}=\frac{R}{c_{v}} Z_{v T}\left(\frac{T_{r}}{v_{r}}\right)^{2}
$$

Based on this statement an analysis of the stability criterion for the ORC fluids in the dense vapour domain has been examined and on the saturation line.

These results are presented in the Figs. 3-5 and in the Tables 2 and 3. A nonlinear behaviour and very low thermodynamic stability of dense gas can be observed in the high-pressure region of the little superheated vapour. In the Fig. 3 the reduced stability $\bar{D}$ of R1234zeZ dense vapour is shown for the range of temperatures $424<T_{r}<$ $430 \mathrm{~K}$ and pressures $0.85<p_{r}<1.15$. Isoclines of constants $\bar{D}$ exhibit the local minima and the saddle points in the vicinity of saturated vapour state.

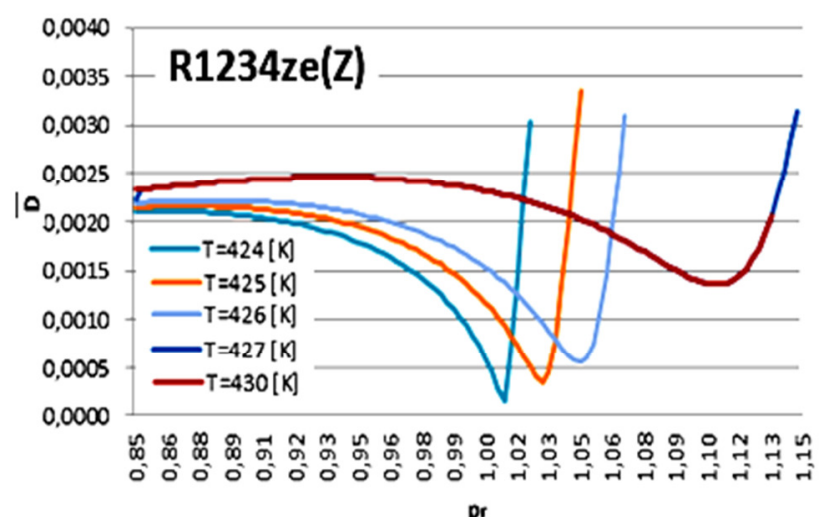

Fig. 3. Reduced thermodynamic stability D of R-1234ze(Z). 
The similar nonlinearities - 'quasispinodals' [10,11], in the reduced stability behaviour are observed for the R134a, see Fig.4 at the supercritical temperatures and the high-pressure range of state parameters.

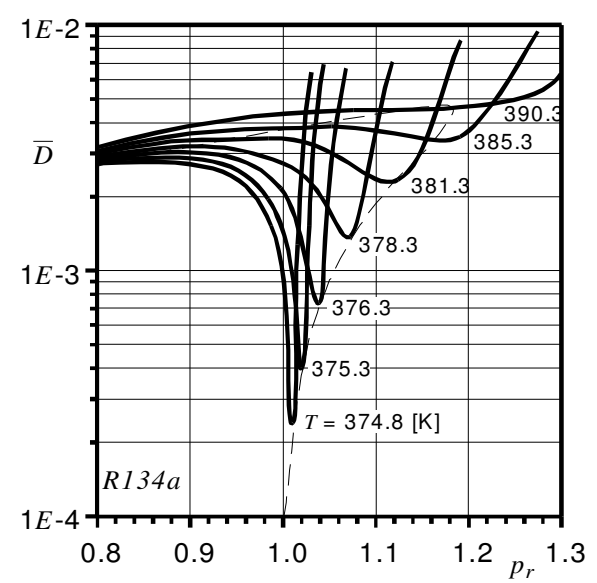

Fig. 4. Stability criterion $\bar{D}$ vs accentric factor $\omega$ of ORC fluids.

In the Fig.5, relation between reduced thermodynamic stability criterion $\bar{D}$ on the saturation line $x=1$, has been ranked according to the accentric factor $\omega$. These data consist three important points on the saturated vapour line

- for the temperature corresponding to $h "=\max$,

- for the temperature corresponding to $s "=\max$,

- at the reduced temperature $T_{r}=0.7$.

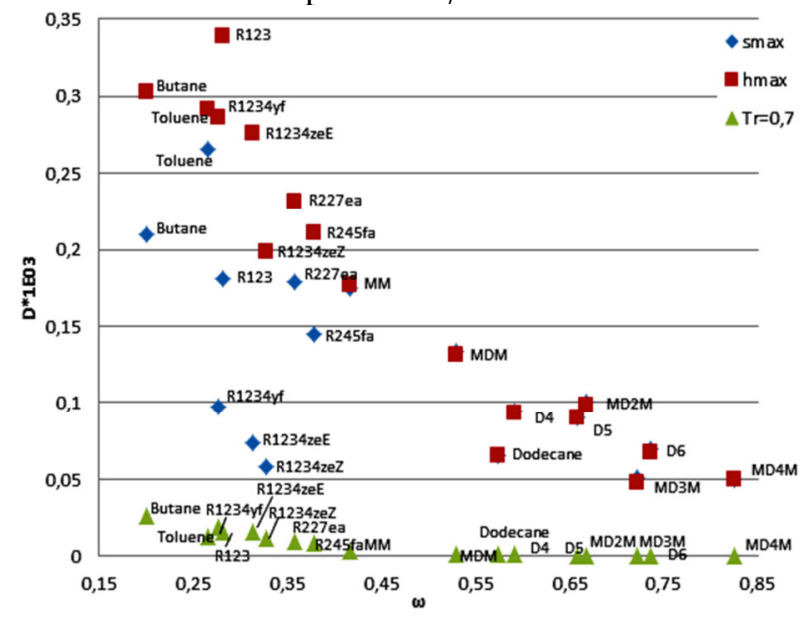

Fig. 5. Stability criterion $\bar{D}$ vs accentric factor $\omega$ of ORC fluids.

It is easy to observe that for a value of reduced temperature $T_{r}=0.7$, the stability criterion $\bar{D}$ on the vapour-liquid boundary line is nearly zero for highmolecular mass and complex fluids such siloxanes. For the hydrocarbons as Butane, Toluene, and its derivatives R123, R227ea, R245fa, and the HFO group R1234ze, it is higher and also positive $\left(\bar{D}=0.14 \div 0.23 \cdot 10^{-3}\right)$. Regarding the behaviour of stability criterion of fluids for the conditions of enthalpy maxima $h^{\prime \prime}{ }_{\max }$ one observes nearly linear dependence of $\bar{D}$ respect to the $\omega$ factor. Only the maxima of saturated vapour entropy $s^{\prime \prime}{ }_{\text {max }}$ for all ORC fluids (isentropic or dry fluids) do not exhibit any regular variation according to its Pitzer's criterion $\omega$. It should be noticed that the stability conditions $\bar{D}$ for the group ofsiloxanes practically coincide both for the $s^{\prime \prime}{ }_{\text {max }}$ and $h^{\prime \prime}{ }_{\text {max }}$ on the saturated vapour line, see Fig.5, for the MM siloxane. The $h^{\prime \prime}{ }_{\max }$ maxima for all analysed ORC fluids corresponds to higher reduced temperatures than values relating to the $s^{\prime \prime}{ }_{\max }$, (see, Appendix Tables 2 and 3).

These results confirm that in the high-density vapour of refrigerants and ORC fluids several peculiarities in fluid behavior should appear, both in the thermodynamic parameters and compressible flow conditions [5,7,10]. One of the most spectacular effects relates to the flow of so-called BZT fluids $[5,10]$.

\subsection{Real gas similarity parameters in the dense vapour area}

Dense gas flow phenomena are still not well-recognised and its investigation, interpreting and mathematical backgrounds is an important part of many research institutions and industry $[5,10]$. It is caused by rapid growth of interesting in utilization of renewable energy and waste heat energy resources including ORC systems and heat pumps.

However, in order to analyse complex phenomena in dense gas area it is necessary to define some more useful parameters which allow to estimate the real fluid flow parameters much more realistic.

Some of the most important quantities will be shortly introduced in the subsequent part of this paper.

\section{- Generalised isentropic index}

The generalised isentropic index $\chi$ plays the same rule as the fundamental derivative of gas dynamics $\Gamma$, [7], but allows to shows the direct relation between the isentropic indices for real and the case of ideal gas model [10]

$$
\begin{aligned}
& \chi=k_{s}+\frac{p}{k_{s}}\left(\frac{\partial k_{s}}{\partial p}\right)_{s}=2 \Gamma-1 \\
& \text { where } \quad \Gamma=-\frac{v}{2}\left(\frac{\partial^{2} p}{\partial v^{2}}\right)_{s} /\left(\frac{\partial p}{\partial v}\right)_{S} \text {. }
\end{aligned}
$$

It is easy to show that at an ideal gas state [10]

$$
\chi=k_{s}=\gamma=c_{p} / c_{v}, \text { and } \Gamma=(\gamma-1) / 2
$$

The molecular complexity of the fluid and real gas effects in a dense vapour flow can significantly affect the change of these parameters. The graphical representation of $\chi$ and isentropic index $k_{s}$ for refrigerant $\mathrm{R} 134 \mathrm{a}$ is presented in the Fig. 6 and Fig.7. 


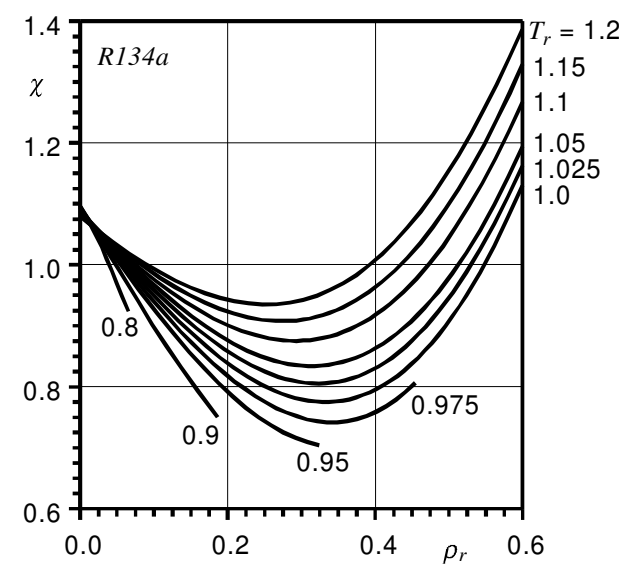

Fig. 6. Generalised isentropic index for R134a [10].

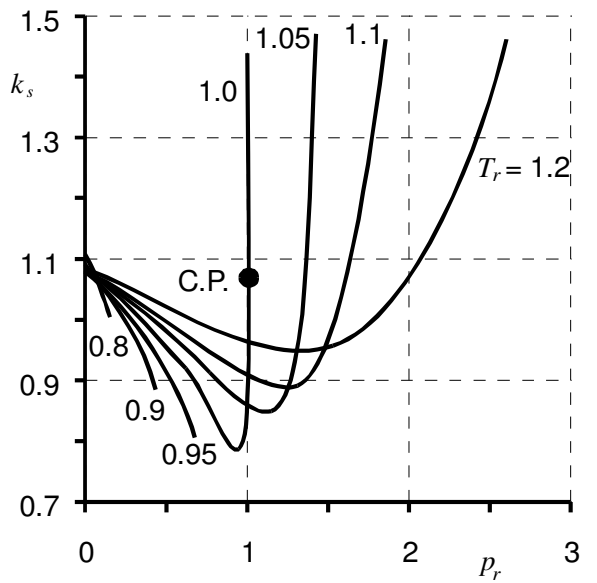

Fig. 7. Isentropic index for R134a [10].

The parameter $\chi$ in the R134a superheated vapour, Fig.6 exhibits a region of its minima in the temperature range $0.8<T_{r}<1.2$, and as the local isentropic index $k_{s}$ can be smaller than one. It means that at the expansion of vapour from the transcritical parameters the variation of state parameters is absolutely different from the classical ideal gas model. When the $\chi$ falls below one, the local speed of sound increases through an isentropic expansion and reduces through isentropic compression [7].

A simple graphical comparison of these three isentropic exponents in the dense gas area are given in the Fig.8. It is visible, that along an isentrope $s=1.71 \mathrm{~kJ} / \mathrm{kgK}$, function $\chi$ varies almost similar as the exponent $k_{s}$, but the Poisson ratio $\gamma$ exhibit an opposite tendency. These nonlinearities indicate a possible local extrema in the variation of Mach Number $M a$ at the isentropic expansion of dense vapour. The exponents $k_{s}$ and $\chi$, as opposed to a Poisson ratio $\gamma$, take values less than unity and pass through a minimum, changing the sign of the derivative.

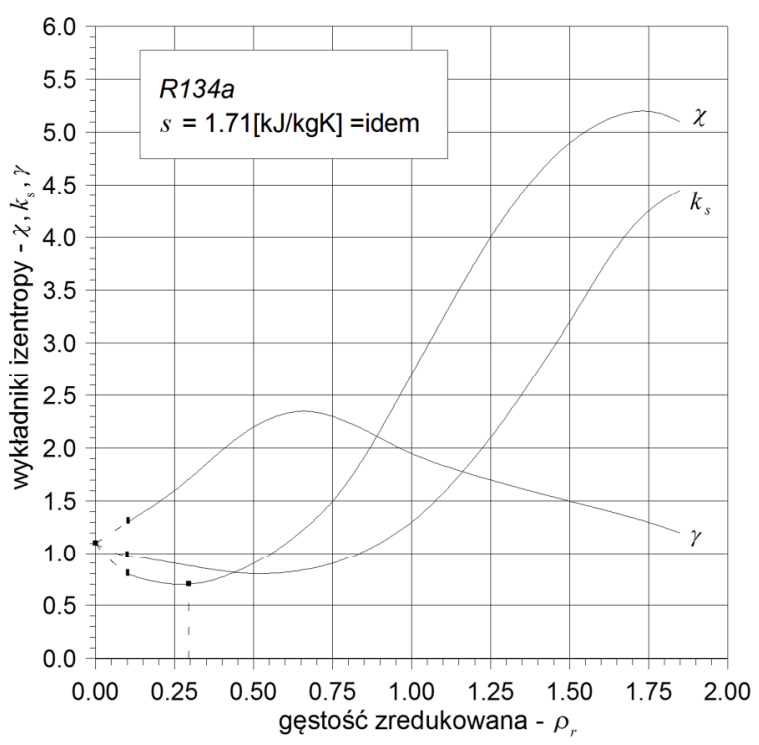

Fig. 8. Comparison the isentropic indices for R134a [11].

\section{- The Grüneisen parameter}

The Grüneisen parameter is another valuable parameter describing peculiarities of the dense fluids $[8,10]$.

From its definition and using the VCD method one obtain

$$
\Phi=-\frac{v}{T}\left(\frac{\partial T}{\partial v}\right)_{S} \equiv \frac{v}{c_{v}}\left(\frac{\partial p}{\partial T}\right)_{v}=\frac{R}{c_{v}} z_{T v}=(\gamma-1) \frac{z_{v T}}{z_{T v}}
$$

It is apparent from the eq. (11), that this parameter is always positive in the one-phase (vapour) region but can be very small in the high-density area, where the VSD's ratio is close to $0.15 \div 0.35$ and the Poisson ratio $\gamma$ goes to the unity, compare data in the Fig. 8.

It should be also mentioned, that at the thermodynamic critical point the Grüneisen parameter has a finite value, and is characteristic for each pure fluid.

\section{- The Thompson function}

From the differential form of Bernoulli equation $[5,7,10]$

$$
\frac{d M a}{M a}=\frac{d \rho}{\rho} J
$$

where

$$
J=-\frac{1}{M a^{2}}\left(1-\frac{\chi-1}{2} M a^{2}\right)
$$

is so-called the 'Thompson function' in the compressible flow [8]. It plays a crucial rule in an analysis of retrograde expansion of dense gases (BZT fluids), such siloxanes and many other refrigerants [5,7]. The Thomson function is always negative for an ideal gas, where $\chi=c_{p} / c_{v}>1$. If only the generalized isentropic exponent $\chi$ is less than one, (see, Fig. 8), this function can be positive and the expansion of the gas will be accompanied by vanishing the shock wave phenomena when fluid-flow process is passing through the sonic point $M a=1$, see, $[5,8,11]$. Such case corresponds to the top part of stagnation temperature $T_{0}$ in the Fig. 9 for an isentropic expansion of R134a. 


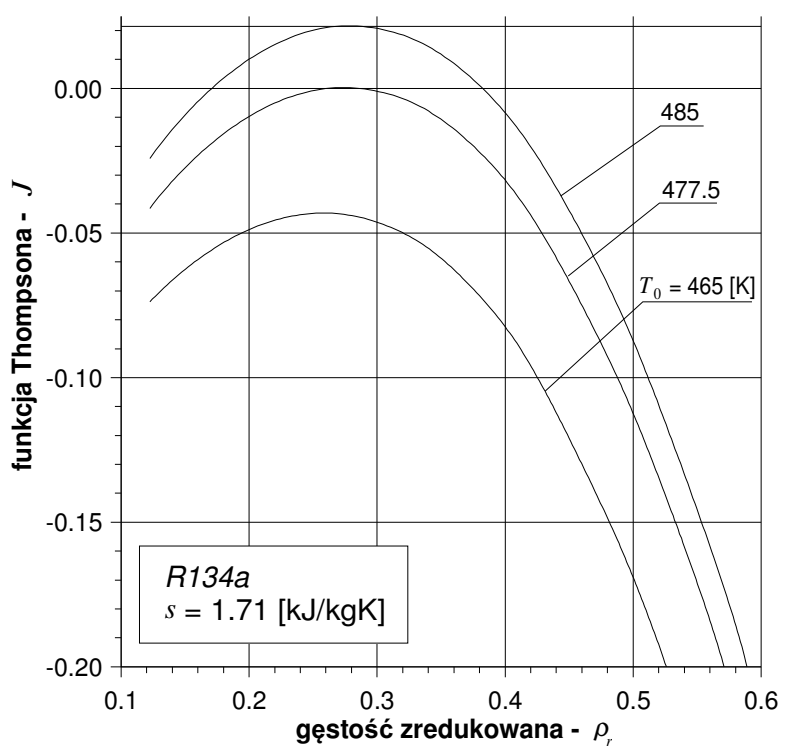

Fig. 9. Thompson function $J$ for R134a [11].

This unique case of possible retrograde expansion for the 'isentropic' fluid R134a is presented in the Fig.10. The non-monotonic change of Mach Number respect to the descending of fluid density $M a=f\left(\rho / \rho_{0}\right)$ is observed for the stagnation temperature $T_{0}=485 \mathrm{~K}$.

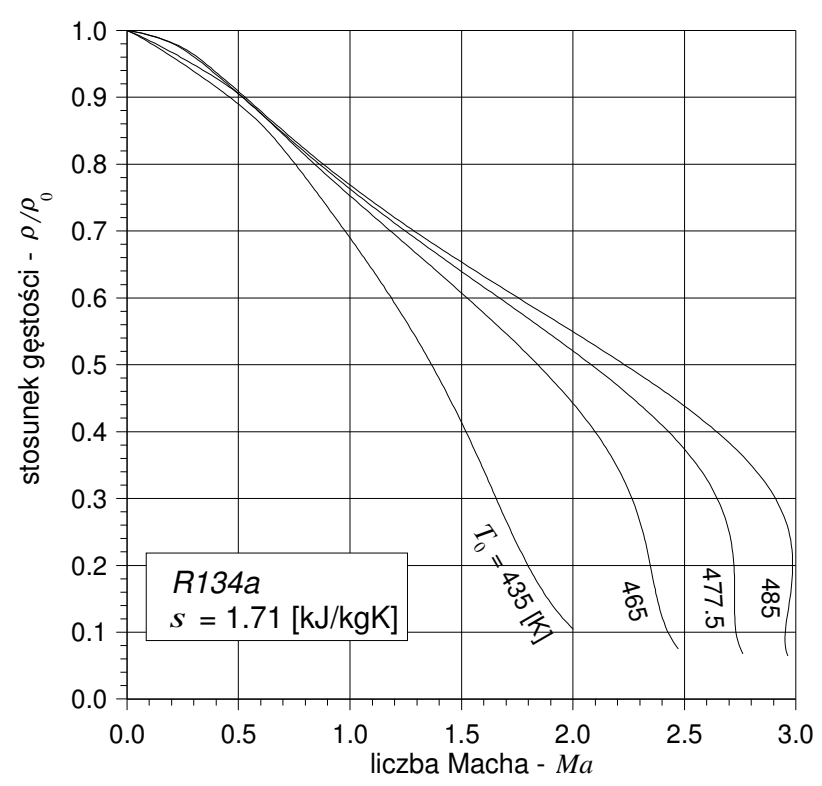

Fig. 10. Possible retrograde expansion of R134a [11].

Therefore, the refrigerant R134a with molecular mass $M$ $=100 \mathrm{~g} / \mathrm{mol}$, and other 'isentropic' fluids should be classified as the media at the frontier of the limiting conditions for 'normal' fluids. All fluids with the large molecular mass $M>100 \mathrm{~g} / \mathrm{mol}$ and relative specific heat $c_{p} / R>10$ must be treated as the real BZT fluids.

\section{Appendix}

- VCD formalism and its application
One of the suggested methods which can be effectively used to analysis of real gas effects is the method of Virial Compressibility Derivatives (VCD), [11]. Based on the Maxwell thermodynamic relations and the Legendre transformation a pair of independent VCD's is given [11]

$$
\begin{aligned}
& z_{T v}=z+T\left(\frac{\partial z}{\partial T}\right)_{v}=z+T_{r}\left(\frac{\partial z}{\partial T_{r}}\right)_{v r} \\
& z_{v T}=z-v\left(\frac{\partial z}{\partial v}\right)_{T}=z-v_{r}\left(\frac{\partial z}{\partial v_{r}}\right)_{T r}
\end{aligned}
$$

where $z=p v /(R T)$ is the compressibility factor. All the above given functions can be directly obtained from a particular form of an EOS for two independent variables temeperature $T$ and specific volume $v$. These functions express the ratio of partial derivatives

$$
\begin{aligned}
& z_{T v} \equiv\left(\frac{\partial p}{\partial T}\right)_{v} /\left(\frac{\partial p^{i d}}{\partial T}\right)_{v} \\
& z_{v T} \equiv\left(\frac{\partial p}{\partial v}\right)_{T} /\left(\frac{\partial p^{i d}}{\partial v}\right)_{T}
\end{aligned}
$$

where $p^{\text {id }}=p(T, v)=R T / v$, corresponds to an ideal gas model, and subsequntly the value of compressibility factor $z$ and the VCD's are equal to one.

In the case of pure fluid, these functions can be also defined for another two independent state variables, for example, if $z=z(T, p)$, we can define [11]

and

$$
z_{T p}=z+T\left(\frac{\partial z}{\partial T}\right)_{p}=z+T_{r}\left(\frac{\partial z}{\partial T_{r}}\right)_{p r}
$$

$$
z_{p T}=z-p\left(\frac{\partial z}{\partial p}\right)_{T}=z-p_{r}\left(\frac{\partial z}{\partial p_{r}}\right)_{T r}
$$

We should notice, that according to chain rule between partial derivatives [10], their linkage relations are [11]

$$
z_{p T} z_{v T}=z^{2} ; \quad z_{T v} / z_{v T}=z_{T p} / Z
$$

Based on the VCD approach, the most important fluid parameters such isentropic index, velocity of sound, and the thermodynamic stability can be simply expressed [11]

$$
\begin{gathered}
k_{S}=-\frac{v}{p}\left(\frac{\partial p}{\partial v}\right)_{S}=-\frac{c_{p}}{c_{v}} \frac{v}{p}\left(\frac{\partial p}{\partial v}\right)_{T}=\gamma \frac{z_{v T}}{z} \\
a_{s}=\sqrt{-v^{2}\left(\frac{\partial p}{\partial v}\right)_{S}}=\sqrt{z_{v T} \gamma R T} \\
D=-\left(\frac{\partial p}{\partial v}\right)_{S}\left(\frac{\partial T}{\partial s}\right)_{p}=-\frac{T}{c_{v}}\left(\frac{\partial p}{\partial v}\right)_{T}=z_{v T} \frac{R}{c_{v}}\left(\frac{T}{v}\right)^{2}
\end{gathered}
$$

The thermal expansion coefficient $\beta_{p}$ " on the saturation line is directly found from eqns. (15) and the chain rule

$$
\beta_{p}^{\prime \prime}=\frac{1}{v^{\prime \prime}}\left(\frac{\partial v^{\prime \prime}}{\partial T}\right)_{p}=\frac{1}{v^{\prime \prime}}\left(\frac{z_{T v}}{z_{v T}}\right)^{\prime \prime}
$$


The Mayer's formula [9] for specific heats difference will be respectively given by

$$
c_{p}-c_{v}=T\left(\frac{\partial p}{\partial v}\right)_{T}^{2}\left(\frac{\partial v}{\partial p}\right)_{T}=R \frac{z_{T v}^{2}}{z_{v T}}
$$

In a limiting ideal gas state all the VCD's as well as the compressibility factor $z$ goes to one.

\section{- Entropy and enthalpy maxima on the right saturation curve}

The condition of saturated vapour entropy maximum $s^{\prime \prime}$ at the saturation condition is given by

$$
\left(\frac{d s}{d T}\right)_{x=1}=\left[1-\frac{T}{c_{p}^{\prime \prime}}\left(\frac{\partial v^{\prime \prime}}{\partial T}\right)_{p}\left(\frac{d p}{d T}\right)_{x=1}\right] /\left(\frac{\partial T}{\partial s}\right)_{p}=0
$$

which can be easily transfomed to the condition

$$
J a \equiv \frac{\Delta h_{l v}}{c_{p}^{\prime \prime}}=\left(\frac{z_{v T}}{z_{T v}}\right)^{\prime \prime}\left(1-\frac{v \prime}{v^{\prime \prime}}\right)
$$

where $J a$ is the Jacob's Number defined based on the heat of vaporisation $\Delta h_{l v}$ and the specific heat of fluid $c_{p}$ on the saturated vapour line. It is easy to find, that the second term in brackets on righ side of the eq. (24) is close to unity, but in opposition the first term is close to $0.25 \div$ 0.35 at the saturation temperatures higher than $T_{r}>0.7$.

Based on Sychev [10], the enthalpy of saturated vapour $h^{\prime \prime}$ max attains its maximum at the point where the JouleThomson coefficient of the dry saturated vapour $\mu_{x=1}$ is equal to its value at the two-phase region (2-ph)

$$
\mu_{x=1}^{\prime \prime}=\mu_{2-p h}
$$

where

$$
\mu_{2-p h}=\left(\frac{d p}{d T}\right)_{x=1}^{-1}, \mu_{x=1}^{\prime \prime}=\frac{v \prime \prime}{c_{p}^{\prime \prime}}\left[\frac{T}{v \prime \prime}\left(\frac{\partial v \prime \prime}{\partial T}\right)_{p}-1\right]
$$

The equation (26) gives a relation between saturation pressure curve shape and the parameters on this line, thus

$$
h_{\text {max }}^{\prime \prime} \stackrel{\text { yields }}{\longrightarrow}\left(\frac{d p}{d T}\right)_{x=1}=\frac{c_{p}^{\prime \prime}}{v^{\prime \prime}\left(\beta_{p}^{\prime \prime} T-1\right)}=\frac{c_{p}^{\prime \prime}}{v^{\prime \prime}\left[\left(\frac{z_{T v}}{z_{v T}}\right)^{\prime \prime}-1\right]}
$$

These two conditions for the saturated vapour entropy and enthalpy maxima have been obtained for the selected groups of ORC fluids based on Refprop package v. 9.1. Values of reduced entropy, the Jacob Number and the reduced stability are corresponding to the proper values of reduced temperatures and pressures, see Table 2.

Table 2. Fluid parameters at $s^{\prime \prime}{ }_{\max }$ on the saturation line.

\begin{tabular}{|l|c|c|c|c|c|}
\hline \multicolumn{1}{|c|}{ Fluid } & $\boldsymbol{T}_{\boldsymbol{r}}$ & $\boldsymbol{p}_{\boldsymbol{r}}$ & $\boldsymbol{s}_{\boldsymbol{m a x}} / \boldsymbol{R}$ & $\boldsymbol{J a} \cdot \mathbf{1 0}^{3}$ & $\overline{\boldsymbol{D}} \cdot \mathbf{1 0}^{\mathbf{3}}$ \\
\hline R123 & 0,9294 & 0,5878 & 31,27 & 171.8 & 2.001 \\
\hline R245fa & 0,9411 & 0,6280 & 29,02 & 130.7 & 0.1451 \\
\hline
\end{tabular}

\begin{tabular}{|l|l|l|l|l|l|}
\hline Butane & 0,9385 & 0,6461 & 17,57 & 139.0 & 0.2098 \\
\hline R227ea & 0,9512 & 0,6843 & 30,85 & 111.4 & 0.1792 \\
\hline Toluene & 0,9690 & 0,7964 & 13,30 & 52.20 & 1.991 \\
\hline Dodecane & 0,9944 & 0,9515 & 21,91 & 9.091 & 0.06577 \\
\hline MM & 0,9870 & 0,9003 & 16,77 & 18.86 & 0.1751 \\
\hline MD2M & 0,9940 & 0,9434 & 22,38 & 7.739 & 0.1000 \\
\hline MDM & 0,9938 & 0,9490 & 20,46 & 11.22 & 0.1335 \\
\hline D4 & 0,9937 & 0,9472 & 21,09 & 8.687 & 0.09446 \\
\hline R1234yf & 0,9015 & 0,4687 & 22,08 & 240.1 & 0.09729 \\
\hline R1234zeE & 0,8915 & 0,4220 & 23,09 & 285.3 & 0.07402 \\
\hline R1234zeZ & 0,8973 & 0,4391 & 24,76 & 238.0 & 0.05780 \\
\hline
\end{tabular}

The presented in Table 2 data compare obtained results for all analysed substances showing that the stability of fluid at the point of entropy maximum is higher for smaller molecular mass refrigerants. The similar behaviour exhibits the Jacob's Number in this limiting point conditions on the temperature-entropy phase diagram.

In the Table 3 similar quantities are collected according to the reduced thermal parameters $T_{r}$ and $p_{r}$, but at the point of $h^{\prime \prime}$ max on the saturation line.

Table 3. Fluid parameters $h_{\max }^{\prime \prime}$ on the saturation line.

\begin{tabular}{|l|c|c|c|c|l|}
\hline \multicolumn{1}{|c|}{ Fluid } & $\boldsymbol{T}_{\boldsymbol{r}}$ & $\boldsymbol{p}_{\boldsymbol{r}}$ & $\boldsymbol{r}_{\boldsymbol{\sigma}} \mathbf{1 0}^{\mathbf{3}}$ & $\boldsymbol{J a} \cdot \mathbf{1 0}^{\mathbf{3}}$ & $\overline{\boldsymbol{D}} \cdot \mathbf{1 0}^{\mathbf{3}}$ \\
\hline R123 & 0,9583 & 0,7348 & -112.8 & 103.5 & 0.9665 \\
\hline R245fa & 0,9612 & 0,7390 & -95.95 & 86.53 & 0.2109 \\
\hline Butane & 0,9621 & 0,7663 & -97.55 & 85.64 & 0.3023 \\
\hline R227ea & 0,9667 & 0,7735 & -85.07 & 76.77 & 0.2319 \\
\hline Toluene & 0,9757 & 0,8373 & -50.70 & 39.72 & 2.019 \\
\hline Dodecane & 0,9944 & 0,9515 & -13.58 & 9.091 & 0.06577 \\
\hline MM & 0,9854 & 0,8893 & -1.923 & 21.37 & 0.1768 \\
\hline MD2M & 0,9943 & 0,9464 & -13.31 & 7.262 & 0.09823 \\
\hline MDM & 0,9942 & 0,9518 & -14.58 & 10.59 & 0.1312 \\
\hline D4 & 0,9940 & 0,9500 & -12.19 & 8.200 & 0.09293 \\
\hline R1234yf & 0,9488 & 0,6838 & -125.0 & 122.8 & 0.2866 \\
\hline R1234zeE & 0,9464 & 0,6652 & -138.3 & 139.3 & 0.2761 \\
\hline R1234zeZ & 0,9481 & 0,6701 & -118.9 & 119.6 & 0.1991 \\
\hline
\end{tabular}

Based on the arguments presented in the sub-section 2.2 it is easy to find that all discussed ORC fluids show in the point of enthalpy extreme negative value of retrogradicity condition $\tilde{r}_{\sigma}$, see equation (3). It should be noticed that for dodecane, a point of maximum entropy coincides with the point of enthalpy maximum. For all other fluids, the stability criterion and the Jacob's Number are of the same order, although they are much higher for the commonly used refrigerants in comparison to the siloxanes. It cofirms, that complex molecules of the fluid means the 
less stable substance which can exhibit many nonlinearities in the thermal-flow processes.

\section{Conclusions}

- All of the analysed fluids have high-molecular complexity, and their behavior is still not well understood. Their choice as ORC fluids is still based only based on the limited data. However, all expected improvements in the performance and efficiency of their components can be attained only by using more complete on the real properties of working media.

- Dense gases are different from their ideal-gas counterparts, because of the different volumetric relations, leading to quantitative deviations in many thermal and fluid-flow parameters. In addition, for fluids that are complex enough, the dependence of the variation selected parameters on the saturation line exhibits some important regularities.

- Furthermore, at the isentropic flow all quantities such as speed of sound and other process indices in the dense-gas region are strongly nonlinear, leading also to qualitative differences in the fluid dynamics.

- An analysis of selected ORC fluids confirmed the main assumptions regards the imposed low thermodynamic stability region on the flow and thermal peculiarities.

- An application of the Virial Compressibility Derivatives (VCD) method allows to simplify and improve an analysisof the complex thermalflow processes in real gases and vapours. It can be used based on the particular form of thermal or fundamental EOS after selection of suitable pair of such parameters and using the Legendre transformation [10,11].

- All new results have been obtained by using the Refprop v.9.1 software package [6] and the VCD analytical tool [11]. Obtained data are based on the reference EOS's for all investigated ORC fluids.

\section{Nomenclature}

$\begin{array}{ll}a_{s} & \text { - velocity of sound, } \mathrm{m} / \mathrm{s} \\ c_{p}, c_{v} & \text { - specific heats, } \mathrm{J} / \mathrm{kgK} \\ D & \text { - thermodynamic stability, } \mathrm{kg}^{2} \mathrm{~K}^{2} / \mathrm{m}^{6} \\ J & \text { - Thompson function } \\ J a & \text { - Jacobs Number } \\ H & \text { - specific enthalpy, } \mathrm{kJ} / \mathrm{kg} \\ k_{s} & \text { - isentropic index } \\ M & \text { - molecular mass, } \mathrm{g} / \mathrm{mol} \\ M a & \text { - Mach Number } \\ p & \text { - pressure, Pa } \\ R & \text { - individual gas constants, } \mathrm{J} /(\mathrm{kgK}) \\ r_{\sigma} & \text { - retrogradicity parameter, eq. (3) } \\ S & \text { - specific entropy, } \mathrm{kJ} /(\mathrm{kg} \mathrm{K}) \\ T & \text { - temperature, } \mathrm{K}\end{array}$

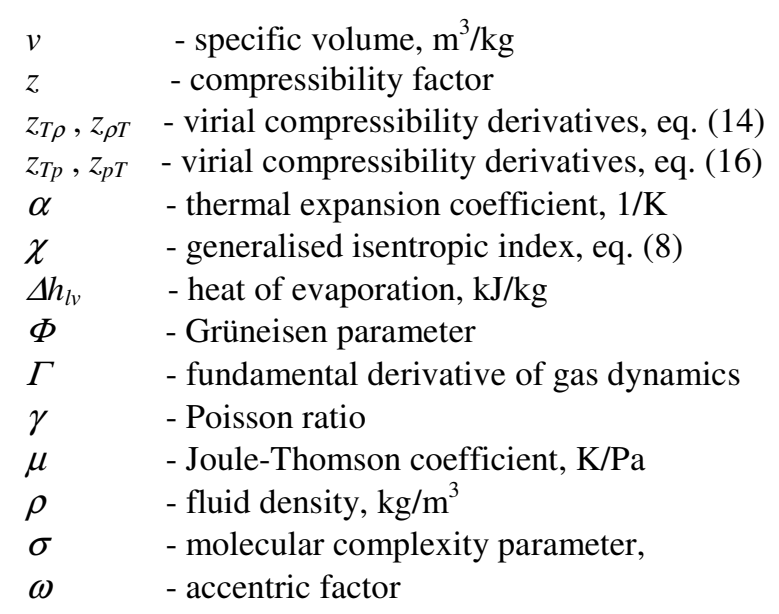

\section{Indices}

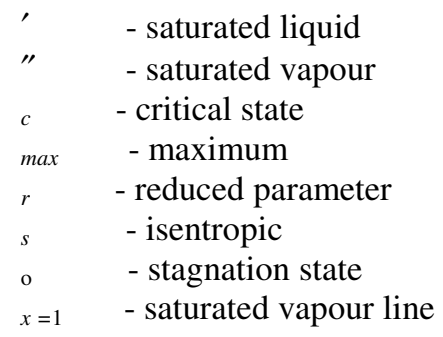

\section{References}

1. B. Saadatfar, R. Fakhrai, T. Franson; Waste heat recovery Organic Rankine cycles in sustainable energy conversion: A state-of-the-art review, JMES 1,1 (2013): 161

2. B.T. Liu, K.H. Chien, C.C. Wang; Effect of working fluids on organic Rankine cycle for waste heat recovery, Energy 29 (2004): 1207

3. J. Bao, L. Zao; A review of working fluid and expander selection for organic Rankie cycle, Renewable \& Sustainable Energy Rev. 24 (2013): 325

4. C. Invernizzi; Closed Power Cycles Thermodynamic Fundamentals and Applications, Lecture Notes in Energy 11, (Springer, 2013)

5. P. Colonna, A. Guardone, N. R. Nannan, Siloxanes: a new class of candidate Bethe-Zel'dovich-Thompson fluids, ' Phys. Fluids, 19, (2007): 086102

6. E.W. Lemmon;M.L. Huber;M.O. McLinden, NIST Standard Reference Database 23: Reference Fluid Thermodynamic \& Transport Properties - REFPROP, V. $9.1(2013)$

7. A.P.S. Wheeler, J. Ong ; Role of dense gas dynamics on organic Rankine cycle turbine performance, ASME Transactions., J. Eng. Gas Turb. \& Power, 135, (Oct. 2013) : 102603

8. P.A. Thompson; Compressible-Fluid Dynamics, (McGraw-Hill, NY, 1988)

9. A. Bejan, Advanced Engineering Thermodynamics, $3^{\text {rd }}$ ed., (J.Wiley, NY, 2006)

10. V.V. Sychev; The Differential Equations of Thermodynamics, $2^{\text {nd }}$ ed., (Hemisphere Publ., 1991)

11. J. Gorski; Modeling of real gas properties and its thermal-flow processes (in Polish), (Oficyna Wyd. Politechniki Rzeszowskiej, Rzeszow, 1997) 